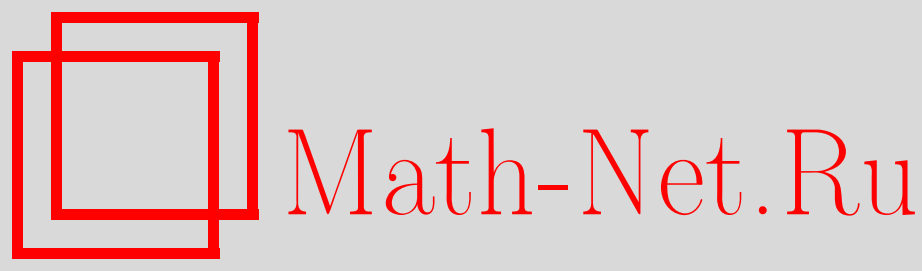

М. В. Либанов, В. А. Рубаков, Космологические возмущения плотности в теории конформного скалярного поля, ТМФ, 2012, том 170, номер 2, 188-205

DOI: https://doi.org/10.4213/tmf6758

Использование Общероссийского математического портала Math-Net.Ru подразумевает, что вы прочитали и согласны с пользовательским соглашением http://www . mathnet.ru/rus/agreement

Параметры загрузки:

IP: 3.95 .254 .165

26 апреля 2023 г., 14:00:43

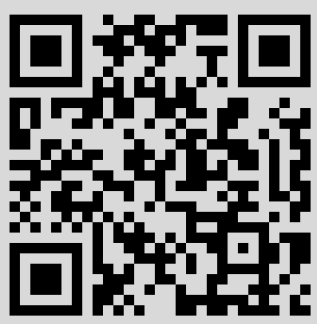




\title{
КОСМОЛОГИЧЕСКИЕ ВОЗМУЩЕНИЯ ПЛОТНОСТИ В ТЕОРИИ КОНФОРМНОГО СКАЛЯРНОГО ПОЛЯ
}

\begin{abstract}
Рассматривается сценарий, в котором первичные космологические скалярные возмущения генерируются благодаря комплексному конформному скалярному полю, скатывающемуся по отрицательному потенциалу четвертой степени. Первоначально эти возмущения являются возмущениями фазы поля, которые затем переходят в адиабатические возмущения плотности. Потенциально опасным свойством этого сценария является наличие возмущений радиальной компоненты поля, имеющих красный спектр мощности. Показано, однако, что в линейном порядке по малому параметру - константе самодействия - инфракрасные эффекты могут быть полностью нивелированы путем надлежащего переопределения поля. Вычислена статистическая анизотропия, присущая модели благодаря наличию длинноволновых возмущений радиальной компоненты поля. В линейном порядке по константе самодействия инфракрасные эффекты не сказываются на статистической анизотропии. Они проявляются только в квадратичном порядке по константе самодействия, слабо (логарифмически) усиливая соответствующий вклад в статистическую анизотропию. Получающаяся в результате статистическая анизотропия является комбинацией большого вклада, который, однако, уменьшается с ростом импульса, и не зависящего от импульса неусиленного вклада.
\end{abstract}

Ключевые слова: альтернативы инфляции, теория космологических возмущений, статистическая анизотропия.

\section{1. ВВЕДЕНИЕ}

Первичные космологические скалярные возмущения во Вселенной обладают двумя основными свойствами - они приближенно гауссовы и их спектр мощности приближенно плоский [1]. Первое свойство указывает на то, что эти возмущения возникают из усиленных вакуумных флуктуаций практически линейного (т. е. слабо взаимодействующего) квантового поля. Действительно, свободное квантовое поле в вакуумном состоянии удовлетворяет теореме Вика, что является определяющим свойством гауссовой случайной величины. С другой стороны, линейная эволюция

* Институт ядерных исследований РАН, Москва, Россия.

E-mail: ml@ms2.inr.ac.ru, rubakov@ms2.inr.ac.ru 
на фоне классического внешнего фонового поля не может генерировать негауссовость. Второе свойство - плоский спектр мощности - подразумевает наличие некоторой симметрии. Наиболее известный кандидат на роль такой симметрии - симметрия пространства де Ситтера по отношению к пространственным растяжениям (дилатациям), дополненным трансляциями времени. Эта симметрия является приближенной симметрией инфляционной вселенной [2], и, действительно, инфляционный механизм генерации скалярных возмущений [3] производит почти плоский спектр мощности. Другой пример такой симметрии - симметрия (уравнений движения) скалярной теории с отрицательным экспоненциальным потенциалом в плоском пространстве-времени. Эта симметрия является симметрией по отношению к пространственно-временным дилатациям, дополненным сдвигами поля. Медленно эволюционирующая вселенная, например вселенная, рожденная в результате столкновения бран [4], или "стартующая" вселенная [5], обладает такой приближенной симметрией, что вновь приводит к почти плоскому спектру мощности [6] (см. также [7] $)^{1)}$.

Еще одна симметрия, которая может быть ответственна за приближенно плоский спектр, была предложена в работе [11]. Она является комбинацией конформной инвариантности и глобальной симметрии. Простейшая модель включает в себя комплексное скалярное поле $\phi$, конформно связанное с гравитацией и достаточно длительный период эволюционирующее в отрицательном потенциале четвертой степени

$$
V(\phi)=-h^{2}|\phi|^{4}
$$

Необходимым условием отсутствия больших нелинейностей на классическом уровне и режима сильной связи на квантовом уровне является ограничение $h<1$. В принципе, как обсуждается в п. 2.2, могут быть и более сильные ограничения на константу $h$. Глобальные преобразования в этой простейшей модели образуют группу $U(1)$, действующую как $\phi \rightarrow e^{i \alpha} \phi$. Предполагается также, что фоновое пространство-время является однородным, изотропным и пространственно плоским, $d s^{2}=a^{2}(\eta)\left(d \eta^{2}-d \mathbf{x}^{2}\right)$. Тогда в силу конформной инвариантности эволюция поля $\chi=a \phi$ не зависит от динамики масштабного фактора и в терминах конформных координат $(\eta, \mathbf{x})$ происходит так же, как и в пространстве-времени Минковского. А именно, мы считаем, что динамика определяется однородным классическим полем $\chi_{\mathrm{c}}(\eta)$, катящимся по отрицательному потенциалу четвертой степени. Поведение этого поля на поздних этапах эволюции полностью определяется конформной инвариантностью. Как отмечается в разделе 2, это катящееся поле генерирует эффективный "горизонт" для возмущений $\delta \chi$ : на ранних этапах линейные возмущения осциллируют в конформном времени как моды свободного безмассового (квантового) скалярного поля ${ }^{2}$, в то время как при поздних временах осцилляционный режим перестает выполняться. Возмущения фазы $\theta=\operatorname{Arg} \phi$ замораживаются в момент

1) Существуют и другие механизмы, способные воспроизвести плоский или практически плоский спектр мощности [8]-[10]. Причем в некоторых случаях не существует какой-либо очевидной симметрии, ответственной за это, т. е. плоскостность спектра мощности является случайной.

2) Здесь предполагается, что стадия скатывания достаточно длительна в конформном времени, так что интересующие нас моды действительно являются "подгоризонтными" при ранних временах. Это предположение нетривиально: конформное скатывание должно происходить в космологическую эпоху, предшествующую стадии Большого взрыва, когда стандартная проблема горизонта по крайней мере формально решена (см. работу [11] и раздел 2). 


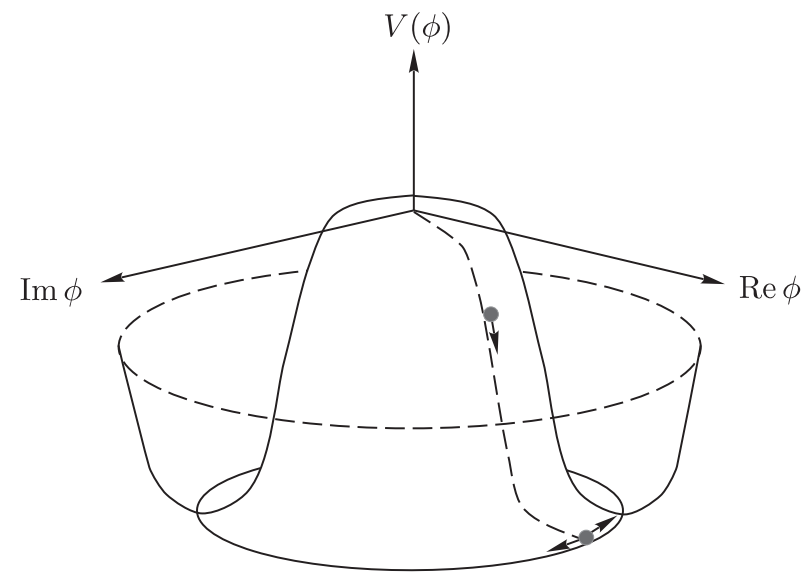

Рис. 1. Скалярный потенциал. Движение шарика демонстрирует эволюцию скалярного поля. Стрелки в конечной точке на дне потенциала показывают возмущения фазы.

выхода за "горизонт", и их спектр мощности становится плоским,

$$
\sqrt{\mathcal{P}_{\delta \theta}}=\frac{h}{2 \pi} .
$$

Это свойство является следствием конформной инвариантности (см. обсуждение в работе [11]).

Далее мы предполагаем, что скалярный потенциал $V(\phi)$ имеет минимум при некотором большом значении $|\phi|$ и что модуль поля $\phi$ в конце концов останавливается в этом минимуме (см. рис. 1). Простейшая возможность дальнейшей эволюции возмущений $\delta \theta$ заключается в предположении, что они являются загоризонтными в обычном смысле в момент, когда конформная стадия закончилась. В этом случае они остаются замороженными ${ }^{3)}$ и их спектр мощности остается плоским. На гораздо более поздних стадиях возмущения фазы преобразуются в адиабатические скалярные возмущения; мы обсуждаем возможные механизмы, ответственные за это, в п. 2.2. Эти механизмы не изменяют спектр мощности, поэтому получающийся в результате спектр мощности адиабатических возмущений остается плоским. Если конформная инвариантность не является точной на стадии скатывания, спектр мощности будет иметь небольшой наклон, который зависит как от величины нарушения конформной инвариантности, так и от эволюции масштабного фактора на стадии скатывания [14].

Специфическим и потенциально опасным свойством модели является то, что модуль скатывающегося поля также имеет возмущения. "Загоризонтные” моды модуля (т. е. радиального направления) имеют красный спектр мощности (см. п. 2.3)

$$
\sqrt{\mathcal{P}_{|\phi|}(k)} \propto k^{-1}
$$

3) Для сжимающейся вселенной это свойство загоризонтных мод выполняется, если доминирующее вещество имеет уравнение состояния с $w>1$. По всей видимости, это условие в любом случае необходимо для жизнеспособности сценария с отскоком [12], [13]. 
Одним из следствий этого является существование возмущений плотности энергии с красным спектром мощности сразу после стадии конформного скатывания, но перед тем, как модуль замораживается в минимуме потенциала $V(\phi)$. Это не является опасным при условии, что плотность энергии поля $\phi$ мала по сравнению с полной плотностью энергии на всех этапах, предшествующих моменту, когда модуль поля останавливается в минимуме своего потенциала. Другими словами, мы предполагаем, что космологическая эволюция на ранних стадиях обусловлена другими видами материи.

Другое следствие заключается в том, что инфракрасные (ИК) моды модуля взаимодействуют с возмущениями фазы и в принципе могут иметь большое влияние на последние. Именно изучение этого влияния и является целью настоящей работы. Для этого мы рассмотрим возмущения фазы в присутствии длинноволновых возмущений модуля, используя градиентное разложение последних. Мы интерпретируем возникающий в нулевом и первом порядках градиентного разложения эффект как локальный сдвиг времени и соответствующее ему лоренцево преобразование фонового поля $\chi_{\text {c }}$. Последнее свойство означает, что скатывающееся фоновое поле практически однородно в локальной системе отсчета, отличающейся от глобальной (космологической) системы отсчета, в которой метрика имеет стандартный вид метрики Фридмана-Робертсона-Уокера. Эта интерпретация дает возможность получить выражение для возмущений фазы, справедливое в первых двух порядках градиентного разложения. Мы покажем, что в линейном порядке по $h$ ИК-эффекты сокращаются: возмущения фазы остаются гауссовыми случайными величинами и имеют плоский спектр мощности (2). Так как красный спектр присущ только $\delta|\phi|$ и $\partial_{i}(\delta|\phi|)$, но не высшим пространственным производным поля $\delta|\phi|$, наш анализ позволяет показать, что ИК-эффекты фактически не приводят к существенным модификациям результатов линейного анализа. Тем не менее это не означает, что можно полностью пренебречь модами $\delta|\phi|$ с большими длинами волн. Те моды, современные длины волн которых превышают хаббловский радиус $H_{0}^{-1}$, индуцируют статистическую анизотропию в возмущениях фазы $\delta \theta$ и, как следствие, в адиабатических возмущениях. В линейном порядке по $h$ статистическая анизотропия генерируется во втором порядке градиентного разложения и, следовательно, свободна от ИК-эффектов. ИК-моды проявляются во втором порядке по константе связи $h$ и ведут к мягкому усилению статистической анизотропии в этом порядке. Соответственно, спектр мощности адиабатических возмущений $\zeta$ имеет следующий вид:

$$
\mathcal{P}_{\zeta}(\mathbf{k})=\mathcal{P}_{0}(k)\left(1+c_{1} h \frac{H_{0}}{k} \widehat{k}_{i} \widehat{k}_{j} w_{i j}-c_{2} h^{2}(\widehat{\mathbf{k}} \mathbf{u})^{2}\right) .
$$

Присутствующая в первом нетривиальном слагаемом величина $w_{i j}$ является бесследовым тензором произвольного вида, нормированным на единицу, $w_{i j} w_{i j}=1$, $\widehat{\mathbf{k}}=\mathbf{k} / k$ - единичный вектор, а $c_{1}$ - константа порядка единицы, ее величина не может быть точно определена из-за космической неопределенности. Как мы уже упоминали, глубокие ИК-моды не влияют на этот член. В последнем слагаемом $\mathbf{u}$ является некоторым единичным вектором, не зависящим от $w_{i j}$, а положительный параметр $c_{2}$ логарифмически усилен благодаря ИК-эффектам. Именно в этом слагаемом ИК-эффекты проявляются впервые. Очевидно, что их влияние подавлено при малых $h$. 
Статистическая анизотропия, описываемая вторым слагаемым в (4), похожа на обычно обсуждаемую в инфляционном контексте [15] и действительно возникающую в некоторых конкретных инфляционных моделях [16]: она не уменьшается с увеличением импульса и имеет фиксированную тензорную форму $(\widehat{\mathbf{k} u})^{2}$ с постоянным вектором u. C другой стороны, первое нетривиальное слагаемое в (4) имеет достаточно общую тензорную структуру и падает с ростом импульса. Это последнее свойство в чем-то напоминает ситуацию, возникающую в космологических моделях с анизотропным расширением перед инфляцией [17]. В общем, статистическая анизотропия (4) может быть достаточно существенной благодаря тому, что по крайней мере в одном из механизмов преобразования возмущений фазы в адиабатические возмущения отсутствуют значительные ограничения на $h$ (см. обсуждение в п. 2.2).

Интересно, что полная статистическая анизотропия является комбинацией большого (порядка $O(h))$ вклада, который, однако, падает с ростом $k$, и меньшего (порядка $O\left(h^{2} \lg \Lambda\right)$, где $\Lambda$ - параметр ИК-обрезания) вклада, не зависящего от $k$ члена. Мы рассматриваем эту особенность как потенциально наблюдаемое характерное свойство нашей модели.

Стоит отметить, что нелинейность скалярного потенциала приводит в предлагаемом сценарии к негауссовости возмущений фазы $\delta \theta$ и, как следствие, адиабатических возмущений помимо той негауссовости, которая может возникнуть на стадии, когда возмущения фазы преобразуются в адиабатические возмущения. В согласии с описанными выше результатами эта негауссовость не зависит от ИК-эффектов в первом нетривиальном порядке по $h$ и может быть полностью вычислена в этом порядке. Однако мы не рассматриваем негауссовость в данной статье, так как градиентное разложение, используемое нами, бесполезно в этом отношении.

Статья имеет следующую структуру. В разделе 2 приведен краткий обзор линейного анализа модели. В разделе 3 мы изучаем влияние ИК-мод модуля поля $\delta|\phi|$ на возмущения фазы $\delta \theta$ в ведущем и следующем порядках градиентного разложения и в линейном порядке по константе самодействия $h$. Статистическая анизотропия, возникающая в следующем порядке, анализируется в разделе 4. В разделе 5 мы подводим итоги.

\section{2. ЛИНЕЙНЫЙ АНАЛИЗ}

На стадии конформного скатывания динамика скалярного поля определяется действием

$$
S[\phi]=\int d^{4} x \sqrt{-g}\left[g^{\mu \nu} \partial_{\mu} \phi^{*} \partial_{\nu} \phi+\frac{R}{6} \phi^{*} \phi-V(\phi)\right],
$$

где скалярный потенциал $V(\phi)$ отрицателен и имеет конформно-инвариантный вид (1). В терминах поля $\chi=a \phi$ действие в конформных координатах имеет тот же вид, что и в пространстве-времени Минковского,

$$
S[\chi]=\int d^{3} x d \eta\left[\eta^{\mu \nu} \partial_{\mu} \chi^{*} \partial_{\nu} \chi+h^{2}|\chi|^{4}\right] .
$$

Уравнение движения имеет вид

$$
\eta^{\mu \nu} \partial_{\mu} \partial_{\nu} \chi-2 h^{2}|\chi|^{2} \chi=0
$$


Пространственно однородные решения стремятся к атрактору при поздних временах

$$
\chi_{\mathrm{c}}(\eta)=\frac{1}{h\left(\eta_{*}-\eta\right)},
$$

где $\eta_{*}$ - произвольный действительный параметр, и мы считаем без потери общности, что это решение действительно. Мы также рассматриваем (6) как фоновое поле. Смысл параметра $\eta_{*}$ заключается в том, что поле $\chi_{\text {с }}$ становится бесконечным при $\eta \rightarrow \eta_{*}$, если скалярный потенциал остается отрицательным потенциалом четвертой степени при любых полях.

2.1. Возмущения фазы. На линейном уровне возмущения фазы и модуля эволюционируют независимо. Мы начнем с изучения возмущений фазы или, для действительного фонового поля (6), возмущений мнимой части $\chi_{2} \equiv \operatorname{Im} \chi$. Они удовлетворяют линеаризованному уравнению

$$
\left(\delta \chi_{2}\right)^{\prime \prime}-\partial_{i} \partial_{i} \delta \chi_{2}-2 h^{2} \chi_{\mathrm{c}}^{2} \delta \chi_{2}=0
$$

где штрих обозначает производную по $\eta$. В явном виде имеем

$$
\left(\delta \chi_{2}\right)^{\prime \prime}-\partial_{i} \partial_{i} \delta \chi_{2}-\frac{2}{\left(\eta_{*}-\eta\right)^{2}} \delta \chi_{2}=0
$$

Пусть $\mathbf{k}$ - конформный импульс возмущений. Важное предположение, которое мы делаем, заключается в том, что стадия скатывания начинается достаточно рано, так что существует промежуток времени, когда выполняется следующее неравенство:

$$
k\left(\eta_{*}-\eta\right) \gg 1
$$

Так как интересные с космологической точки зрения импульсы $k$ так же малы, как современный параметр Хаббла, это неравенство означает, что длительность стадии скатывания в конформном времени больше, чем конформное время, прошедшее, скажем, с момента горячего Большого взрыва до настоящей эпохи. Это возможно только при условии, что стадия горячего Большого взрыва предварялась некоторой другой эпохой, которая решает стандартную проблему горизонта; мы считаем, что обсуждаемый здесь механизм работает именно на этой стадии. Попутно заметим, что последнее свойство присуще большинству, если не всем, механизмов генерации космологических возмущений.

Уравнение (8) в точности совпадает с уравнением для минимально связанного безмассового скалярного поля в пространстве де Ситтера. Тем не менее мы кратко обсудим свойства его решений. На ранних этапах, когда выполняется неравенство $(9), \delta \chi_{2}$ - это свободное безмассовое квантовое скалярное поле,

$$
\delta \chi_{2}(\mathbf{x}, \eta)=\int \frac{d^{3} k}{(2 \pi)^{3 / 2} \sqrt{2 k}}\left(\delta \chi_{2}^{(-)}(\mathbf{k}, \mathbf{x}, \eta) \hat{A}_{\mathbf{k}}+\text { э.c. }\right),
$$

моды которого имеют вид (мы сохраняем зависимость от х для удобства)

$$
\delta \chi_{2}^{(-)}(\mathbf{k}, \mathbf{x}, \eta)=e^{i \mathbf{k x}-i k \eta} .
$$

2 Теоретическая и математическая физика, т. 170, № 2, 2012 г. 
Здесь $\hat{A}_{\mathbf{k}}$ и $\hat{A}_{\mathbf{k}}^{\dagger}$ - операторы уничтожения и рождения, удовлетворяющие стандартным коммутационным соотношениям, $\left[\hat{A}_{\mathbf{k}}, \hat{A}_{\mathbf{k}^{\prime}}^{\dagger}\right]=\delta\left(\mathbf{k}-\mathbf{k}^{\prime}\right)$. Естественно предположить, что вначале поле $\delta \chi_{2}$ находится в вакуумном состоянии.

Скатывающееся внешнее поле $\chi_{c}(\eta)$ создает эффективный "горизонт" для возмущений $\delta \chi_{2}$. Осцилляции (10) заканчиваются, когда соответствующая мода выходит за "горизонт", т. е. при $k\left(\eta_{*}-\eta\right) \sim 1$. Решение уравнения (8) с начальным условием (10) имеет вид

$$
\delta \chi_{2}^{(-)}(\mathbf{k}, \mathbf{x}, \eta)=e^{i \mathbf{k x}-i k \eta_{*}} F\left(k, \eta_{*}-\eta\right),
$$

где

$$
F(k, \xi)=-\sqrt{\frac{\pi}{2} k \xi} H_{3 / 2}^{(1)}(k \xi)
$$

и $H_{3 / 2}^{(1)}$ - функция Ханкеля. В “загоризонтном” режиме при поздних временах, когда $k\left(\eta_{*}-\eta\right) \ll 1$,

$$
F\left(k, \eta_{*}-\eta\right)=\frac{i}{k\left(\eta_{*}-\eta\right)} .
$$

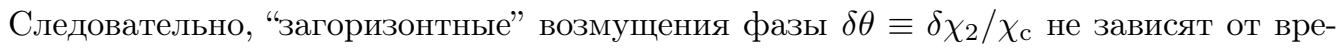
мени,

$$
\delta \theta(\mathbf{x})=\frac{\delta \chi_{2}(\mathbf{x}, \eta)}{\chi_{\mathrm{c}}(\eta)}=h \int \frac{d^{3} k}{4 \pi^{3 / 2} k^{3 / 2}} i e^{i \mathbf{k x}-i k \eta_{*}} \hat{A}_{\mathbf{k}}+\text { э.c. }
$$

Это выражение описывает случайную гауссову величину (ср. [18]), спектр мощности которой дается выражением (2).

2.2. Переход возмущений фазы в адиабатические возмущения. Для полноты изложения мы кратко обсудим два возможных пути перехода возмущений фазы $\delta \theta$ в адиабатические возмущения.

Первый путь заключается в использовании курватонного механизма [19] в применении к псевдо-намбу-голдстоуновскому курватону [20]. Именно, предположим, что $\theta$ в действительности является псевдо-намбу-голдстоуновским полем. В общем случае конформное скатывание заканчивается на склоне потенциала (см. рис. 2).

Поле $\theta$ вместе с его возмущениями остается замороженным до стадии горячего Большого взрыва, когда параметр Хаббла становится сравним с массовым параметром поля. В этот момент поле $\theta$ начинает осциллировать вблизи минимума его потенциала. При условии, что это поле взаимодействует с обычными частицами, его осцилляции в конечном итоге затухают, и запасенная в нем энергия переходит в энергию космической плазмы. Именно на этом этапе возмущения плотности энергии поля $\theta$ конвертируются в адиабатические возмущения. Форма получаемого в результате адиабатического спектра мощности совпадает с формой спектра $\delta \theta$, т. е. в случае точной конформной инвариантности на стадии скатывания спектр является плоским. В общем случае амплитуда адиабатических возмущений порядка

$$
\sqrt{\mathcal{P}_{\zeta}} \sim r \frac{\sqrt{\mathcal{P}_{\delta \theta}}}{\theta_{\mathrm{c}}}=r \frac{h}{2 \pi \theta_{\mathrm{c}}},
$$

где $r$ - отношение плотности энергии поля $\theta$ к полной плотности энергии в момент, когда осцилляции $\theta$ полностью затухают, а $\theta_{\text {c }}$ - расстояние от точки, в которой заканчивается конформное скатывание, до настоящего минимума потенциала. 


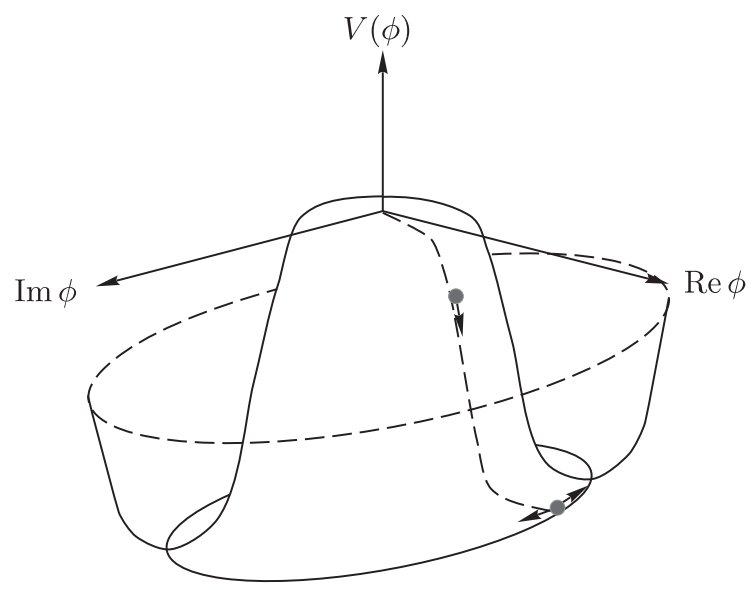

Рис. 2. Скалярный потенциал в псевдо-намбу-голдстоуновском сценарии.

Псевдо-намбу-голдстоуновский механизм также генерирует негауссовость локального вида в адиабатических возмущениях. Считая в общем случае, что значения фазы в момент завершения конформного скатывания $\theta_{\text {c }} \sim \pi / 2$, из отсутствия негауссовости [1] находим $r \gtrsim 10^{-2}$, так что правильная величина амплитуды получается при

$$
h \lesssim 10^{-2}
$$

Таким образом, сценарий с псевдо-намбу-голдстоуновским механизмом конвертации возмущений фазы в адиабатические возмущения жизнеспособен только для малых значений константы скалярного самодействия.

Другой возможностью является модулированный распад тяжелых частиц [21][23]. Предполагается, что фаза $\theta$ взаимодействует с некоторыми тяжелыми частицами так, что массы и/или ширины последних зависят от $\theta$,

$$
M=M_{0}+\epsilon_{M} \theta \quad \text { и/или } \quad \Gamma=\Gamma_{0}+\epsilon_{\Gamma} \theta .
$$

Предполагается также, что эти частицы выживают в эпоху горячего Большого взрыва до момента, когда они становятся нерелятивистскими и доминантными в космологическом расширении. Когда эти частицы распадаются, возмущения $\theta$ и, следовательно, $M$ и/или Г индуцируют адиабатические возмущения,

$$
\zeta \sim \frac{\delta M}{M}=\frac{\epsilon_{M} \delta \theta}{M_{0}+\epsilon_{M} \theta_{\mathrm{c}}} \quad \text { и/или } \quad \zeta \sim \frac{\delta \Gamma}{\Gamma}=\frac{\epsilon_{\Gamma} \delta \theta}{\Gamma_{0}+\epsilon_{\Gamma} \theta_{\mathrm{c}}} .
$$

Форма спектра мощности адиабатических возмущений снова совпадает с начальной формой спектра $\delta \theta$.

Механизм модулированного распада также индуцирует негауссовость в адиабатических возмущениях. Однако, если зависимость массы или ширины от $\theta$ линейна, как в (16), индуцированная негауссова часть адиабатических возмущений имеет порядок

$$
(\delta M / M)^{2},(\delta \Gamma / \Gamma)^{2} \sim \zeta^{2}
$$


Другими словами, вне зависимости от величины константы $h$, параметр негауссовости достаточно мал (подробнее см. работы [22], [23]), $f_{\mathrm{nl}} \sim 1$, что находится в хорошем согласии с существующим пределом [1]. Таким образом, механизм модулированного распада сам по себе не налагает каких-либо ограничений на константу $h$.

2.3. Возмущения модуля. Вернемся к стадии конформного скатывания и рассмотрим радиальные возмущения поля - возмущения модуля $\chi$ или, принимая во внимание наше соглашение о действительности фонового поля $\chi_{\mathrm{c}}$, возмущения действительной части модуля $\chi_{1} \equiv \operatorname{Re} \chi$. На линейном уровне они удовлетворяют уравнению

$$
\left(\delta \chi_{1}\right)^{\prime \prime}-\partial_{i} \partial_{i} \delta \chi_{1}-6 \chi_{\mathrm{c}}^{2} \delta \chi_{1} \equiv\left(\delta \chi_{1}\right)^{\prime \prime}-\partial_{i} \partial_{i} \delta \chi_{1}-\frac{6}{\left(\eta_{*}-\eta\right)^{2}} \delta \chi_{1}=0
$$

Его решение, стремящееся к надлежащим образом нормированной моде свободного квантового поля при $k\left(\eta_{*}-\eta\right) \rightarrow \infty$, имеет вид

$$
\delta \chi_{1}=e^{i \mathbf{k x}-i k \eta_{*}} \frac{1}{4 \pi} \sqrt{\frac{\eta_{*}-\eta}{2}} H_{5 / 2}^{(1)}\left[k\left(\eta_{*}-\eta\right)\right] \widehat{B}_{\mathbf{k}}+\text { э.c. },
$$

где $\widehat{B}_{\mathbf{k}}, \widehat{B}_{\mathbf{k}}^{\dagger}$ - другой набор операторов уничтожения и рождения. На поздних этапах, когда $k\left(\eta_{*}-\eta\right) \ll 1$ (“загоризонтный" режим), имеем

$$
\delta \chi_{1}=e^{i \mathbf{k x}-i k \eta_{*}} \frac{3}{4 \pi^{3 / 2}} \frac{1}{k^{5 / 2}\left(\eta_{*}-\eta\right)^{2}} \widehat{B}_{\mathbf{k}}+\text { э.c. }
$$

Следовательно, “загоризонтные” возмущения модуля имеют красный спектр (3).

Зависимость $\delta \chi_{1} \propto\left(\eta_{*}-\eta\right)^{-2}$ имеет естественную интерпретацию в терминах локального сдвига параметра “конечного времени" $\eta_{*}$. Действительно, для фонового поля (6) сумма $\chi_{\mathrm{c}}+\delta \chi_{1}$, т. е. радиальное поле, включающее возмущение, является линеаризованной формой функции

$$
\chi_{\mathrm{c}}\left[\eta_{*}(\mathbf{x})-\eta\right]=\frac{1}{h\left[\eta_{*}(\mathbf{x})-\eta\right]},
$$

где

$$
\eta_{*}(\mathbf{x})=\eta_{*}+\delta \eta_{*}(\mathbf{x})
$$

И

$$
\delta \eta_{*}(\mathbf{x})=\frac{3 h}{4 \pi^{3 / 2}} \int \frac{d^{3} k}{k^{5 / 2}}\left(e^{i \mathbf{k x}-i k \eta_{*}} \widehat{B}_{\mathbf{k}}+\text { э.c. }\right) .
$$

Так что, как это следует из (17), (18), ИК-моды модифицируют эффективное фоновое поле, заменяя параметр "конечного времени" $\eta_{*}$ на случайное, медленно меняющееся в пространстве поле ${ }^{4)}$. Ясно, что этот факт остается справедливым вне рамок линейного приближения: как только пространственный масштаб изменения $\chi_{1}(\mathbf{x}, \eta)$ превышает размер "горизонта", градиенты в уравнении (5) становятся пренебрежимо малыми, и решения полного нелинейного уравнения при поздних временах локально имеют одну и ту же форму (6) по модулю медленного изменения $\eta_{*}$ в пространстве.

\footnotetext{
4) Имеются поправки к (17) порядка $\partial_{i} \partial_{j} \eta_{*}(\mathbf{x})$ и $\left[\partial_{i} \eta_{*}(\mathbf{x})\right]^{2}$, см. п. 4.1, 4.2.
} 
Стоит отметить, что ИК-моды дают вклад как в поле $\delta \eta_{*}(\mathbf{x})$ само по себе, так и в его пространственную производную. Вклад мод, являющихся сегодня загоризонтными, т. е. имеющих импульсы $k \lesssim H_{0}$, в изменение пространственной производной поля имеет вид

$$
\left\langle\partial_{i} \eta_{*}(\mathbf{x}) \partial_{j} \eta_{*}(\mathbf{x})\right\rangle_{k \lesssim H_{0}}=\delta_{i j} \frac{3 h^{2}}{4 \pi} \int_{k \lesssim H_{0}} \frac{d k}{k}=\delta_{i j} \frac{3 h^{2}}{4 \pi} \ln \frac{H_{0}}{\Lambda},
$$

где $\Lambda$ - параметр ИК-обрезания, который характеризует наше пренебрежение динамикой в начале стадии конформного скатывания.

\section{3. ВЛИЯНИЕ ИНФРАКРАСНЫХ РАДИАЛЬНЫХ МОД НА ВОЗМУЩЕНИЯ ФАЗЫ: ПЕРВЫЙ ПОРЯДОК ПО $h$}

Основная цель настоящей статьи заключается в изучении того, как взаимодействие с радиальными ИК-модами изменяет свойства возмущений фазы $\delta \theta$. Для этого мы рассмотрим возмущения мнимой части $\delta \chi_{2}$, длины волн которой много меньше, чем масштаб изменения модуля в пространстве. При этом благодаря разделению масштабов все еще можно рассматривать возмущения $\delta \chi_{2}$ в линейном приближении, но уже во внешнем поле (17).

Так как нас интересует ИК-часть $\eta_{*}(\mathbf{x})$, мы будем использовать градиентное разложение, рассматривая область около начала координат:

$$
\eta_{*}(\mathbf{x})=\eta_{*}(0)-v_{i} x_{i}+\cdots,
$$

где

$$
v_{i}=-\left.\partial_{i} \eta_{*}(\mathbf{x})\right|_{\mathbf{x}=0},
$$

а многоточие обозначает члены более высокого порядка по х. Важно, что поле $\partial_{i} \partial_{j} \eta_{*}(\mathbf{x})$ имеет голубой спектр, в отличие от $\eta_{*}(\mathbf{x})$ и $\partial_{i} \eta_{*}(\mathbf{x})$, так что основное влияние ИК-мод обусловлено первыми двумя членами градиентного разложения, явно выписанными в (21). В этом разделе мы будем работать в первом порядке разложения по производным. Кроме того, мы предполагаем в дальнейшем, что

$$
|\mathbf{v}| \ll 1,
$$

и в этом разделе мы пренебрегаем поправками порядка $\mathbf{v}^{2}$. Разложение по $|\mathbf{v}|$ оправданно в силу того, что $\mathbf{v}(\mathbf{x})$ имеет плоский спектр мощности, поэтому спектр мощности $\mathbf{v}$ имеет порядок $h^{2}|\ln \Lambda|(\Lambda$ - параметр ИК-обрезания) и мало для малых $h$ и не слишком больших значений $|\ln \Lambda|$. Другими словами, разложение по $|\mathbf{v}|$ является разложением по $h$ с учетом ИК-логарифмов. Анализ главного эффекта, возникающего в порядке $\mathbf{v}^{2}$, мы проведем в п. 4.2.

Сохраняя только два члена в разложении (21), вместо уравнения (8) мы получаем

$$
\left(\delta \chi_{2}\right)^{\prime \prime}-\partial_{i} \partial_{i} \delta \chi_{2}-\frac{2}{\left[\eta_{*}(0)-\mathbf{v} \mathbf{x}-\eta\right]^{2}} \delta \chi_{2}=0
$$

Мы видим, что знаменатель в выражении для фонового поля

$$
\chi_{\mathrm{c}}=\frac{1}{h\left[\eta_{*}(0)-\eta-\mathbf{v x}\right]}
$$


содержит комбинацию $\eta_{*}(0)-(\eta+\mathbf{v x})$. Это можно интерпретировать как локальный сдвиг времени и преобразование Лоренца первоначального фонового поля (6): эффективное фоновое поле является однородным в системе отсчета (в конформных координатах), отличающейся от космологической системы отсчета, в которой метрика пространственно однородна. Заметим, что в этом приближении поле (24) является решением уравнения (5). Наша интерпретация предполагает, что решения уравнения (23) могут быть получены путем сдвига времени и преобразования Лоренца из исходного решения (11), (12). Действительно, легко увидеть, что в первом порядке по $\mathbf{v}$ решение уравнения (23), удовлетворяющее начальным условиям (10), имеет вид

$$
\delta \chi_{2}^{(-)}(\mathbf{k}, \mathbf{x}, \eta)=e^{i \mathbf{q}(\mathbf{x}+\mathbf{v} \eta)-i q \eta_{*}(0)} F\left(q, \eta_{*}(0)-\eta-\mathbf{v x}\right),
$$

где функция $F$ по-прежнему определена в $(12)$, а лоренц-преобразованный импульс

$$
\mathbf{q}=\mathbf{k}+k \mathbf{v}, \quad q=|\mathbf{q}|=k+\mathbf{k v},
$$

при этом подразумевается, что члены порядка $\mathbf{v}^{2}$ должны быть отброшены. Решение (25) может быть представлено в следующей форме:

$$
\delta \chi_{2}^{(-)}(\mathbf{k}, \mathbf{x}, \eta)=e^{i \mathbf{k x}-i k \eta_{*}(\mathbf{x})-i \mathbf{k} \mathbf{v}\left[\eta_{*}(\mathbf{x})-\eta\right]} F\left(q, \eta_{*}(\mathbf{x})-\eta\right),
$$

где $v_{i}=-\partial_{i} \eta_{*}(\mathbf{x})$. Этот вид, справедливый до первого порядка градиентного разложения и до линейного порядка по $\mathbf{v}$ включительно, не зависит от выбора начала координат и может быть использован при произвольных х. Мы рассмотрим поправки порядка $\partial_{i} \partial_{j} \eta_{*}(\mathbf{x})$ и $\mathbf{v}^{2}$ к этому решению в п. 4.1 и п. 4.2 соответственно.

Из выражений (24) и (25) (или, что то же самое, (17) и (27)) следует, что возмущения фазы снова замораживаются при $k\left[\eta_{*}(\mathbf{x})-\eta\right] \rightarrow 0$ и принимают вид

$$
\delta \theta(\mathbf{x})=\frac{\delta \chi_{2}(\mathbf{x})}{\chi_{\mathrm{c}}(\mathbf{x})}=i \int \frac{d^{3} k}{\sqrt{k}} \frac{h}{4 \pi^{3 / 2} q} e^{i \mathbf{k x}-i k \eta_{*}(\mathbf{x})} \hat{A}_{\mathbf{k}}+\text { э.c. },
$$

где соотношение между $\mathbf{k}$ и $\mathbf{q}$ по-прежнему задается в $(26)$. Напомним, что $\eta_{*}(\mathbf{x})$ является некоторой реализацией случайного ИК-поля. Формула (28) подразумевает, что вплоть до первого порядка градиентного разложения, которым мы ограничиваемся в этом разделе, свойства случайного поля $\delta \theta$ точно такие же, как и у линейного поля (14). Рассмотрим, например, двухточечную корреляционную функцию

$$
\left\langle\delta \theta\left(\mathbf{x}_{1}\right) \delta \theta\left(\mathbf{x}_{2}\right)\right\rangle=\int \frac{d^{3} k}{k} \frac{h^{2}}{16 \pi^{3} q^{2}} e^{i \mathbf{k}\left(\mathbf{x}_{1}-\mathbf{x}_{2}\right)-i k\left[\eta_{*}\left(\mathbf{x}_{1}\right)-\eta_{*}\left(\mathbf{x}_{2}\right)\right]}+\text { k.c. }
$$

Рассматриваемые ИК-моды $\eta_{*}(\mathbf{x})$ имеют импульсы, много меньшие, чем $k$, т. е. пространственные масштабы, на которых поле изменяется, много больше $\left|\mathbf{x}_{1}-\mathbf{x}_{2}\right|$. Следовательно, до первого порядка градиентного разложения мы имеем $\eta_{*}\left(\mathbf{x}_{1}\right)-$ $\eta_{*}\left(\mathbf{x}_{2}\right)=-\mathbf{v}\left(\mathbf{x}_{1}-\mathbf{x}_{2}\right)$, и $\mathbf{v}$ не зависит от $\mathbf{x}$. Таким образом, двухточечная функция имеет вид

$$
\left\langle\delta \theta\left(\mathbf{x}_{1}\right) \delta \theta\left(\mathbf{x}_{2}\right)\right\rangle=\int \frac{d^{3} k}{k} \frac{h^{2}}{16 \pi^{3} q^{2}} e^{i \mathbf{q}\left(\mathbf{x}_{1}-\mathbf{x}_{2}\right)}+\text { к.c. }
$$

Делая замену переменной интегрирования $\mathbf{k} \rightarrow \mathbf{q}$ и учитывая, что мера $d^{3} k / k$ лоренц-инвариантна, получаем

$$
\left\langle\delta \theta\left(\mathbf{x}_{1}\right) \delta \theta\left(\mathbf{x}_{2}\right)\right\rangle=h^{2} \int \frac{d^{3} q}{16 \pi^{3} q^{3}} e^{i \mathbf{q}\left(\mathbf{x}_{1}-\mathbf{x}_{2}\right)}+\text { K.c. },
$$


что в точности совпадает с двухточечной функцией линейного поля (14).

Этот аргумент непосредственно обобщается на многоточечные функции: для данной реализации случайного поля $\eta_{*}(\mathbf{x})$ все корреляторы выражаются в терминах двухточечной функции (29). Другими словами, ИК-эффекты полностью удаляются с помощью переопределения поля,

$$
\hat{\mathcal{A}}_{\mathbf{q}}=e^{-i k \eta_{*}(0)} \sqrt{\frac{k}{q}} \hat{A}_{\mathbf{k}}
$$

где $\mathbf{k}$ и q по-прежнему связаны соотношением (26). Операторы $\hat{\mathcal{A}}_{\mathbf{q}}, \hat{\mathcal{A}}_{\mathbf{q}}^{\dagger}$ подчиняются стандартным коммутационным соотношениям, тогда как в нашем приближении поле (28), записанное в терминах этих операторов, совпадает с линейным полем (14). Таким образом, мы заключаем, что ИК-моды в действительности не опасны, так как они не имеют большого влияния на свойства поля $\delta \theta$.

\section{4. СТАТИСТИЧЕСКАЯ АНИЗОТРОПИЯ}

4.1. Первый порядок по $h$. Продолжим наш анализ в первом порядке по $h$. В этом приближении нетривиальное влияние длинноволновых возмущений $\delta \eta_{*}(\mathbf{x})$ на возмущения фазы и, как следствие, на адиабатические возмущения возникает в первый раз во втором порядке градиентного разложения, т. е. в порядке $\partial_{i} \partial_{j} \eta_{*}$. Сконцентрируемся на влиянии мод $\delta \eta_{*}$, современные длины волн которых превышают современный хаббловский размер. Мы имеем дело только с одной реализацией случайного поля. Следовательно, во втором порядке градиентного разложения величина $\partial_{i} \partial_{j} \eta_{*}$ является просто тензором, постоянным во всей видимой Вселенной. Вычислим статистическую анизотропию, ассоциированную с этим тензором.

С этой целью мы используем теорию возмущений по $\partial_{i} \partial_{j} \eta_{*}$. В первую очередь мы должны найти новое фоновое поле, так как во втором порядке градиентного разложения функция (17) более не является решением уравнения поля (5). Пусть

$$
\chi_{\mathrm{c}}=\chi_{\mathrm{c}}^{(1)}+\chi_{\mathrm{c}}^{(2)}
$$

где

$$
\chi_{\mathrm{c}}^{(1)}=\frac{1}{h\left[\eta_{*}(\mathbf{x})-\eta\right]}
$$

и $\chi_{\mathrm{c}}^{(2)}=O\left(\partial_{i} \partial_{j} \eta_{*}\right)$. Подставляя выражение (31) в уравнение (5) и линеаризуя по $\partial_{i} \partial_{j} \eta_{*}$, получим следующее уравнение для поправки:

$$
\chi_{\mathrm{c}}^{(2) \prime \prime}-\frac{6}{\left(\eta_{*}-\eta\right)^{2}} \chi_{\mathrm{c}}^{(2)}=-\frac{\partial_{i} \partial_{i} \eta_{*}}{h\left(\eta_{*}-\eta\right)^{2}},
$$

где мы снова пренебрегаем членами порядка $\mathbf{v}^{2}$. Ясно, что эта поправка к фоновому полю зависит только от скаляра $\partial_{i} \partial_{i} \eta_{*}$, так что она не вызывает статистической анизотропии. Тем не менее мы будем удерживать эту поправку в дальнейшем.

Общее решение уравнения (33) имеет вид

$$
\chi_{\mathrm{c}}^{(2)}=C_{1}\left(\eta_{*}-\eta\right)^{3}+\frac{C_{2}}{\left(\eta_{*}-\eta\right)^{2}}+\frac{1}{6 h} \partial_{i} \partial_{i} \eta_{*} .
$$


Первый член в правой части не имеет отношения к делу в “загоризонтном" режиме, второй член просто является сдвигом $\eta_{*}$ в выражении (32) для лидирующего порядка, так что нетривиальная поправка дается третьим членом. Комбинация, входящая в уравнение (7) для возмущений мнимой части, теперь имеет вид (мы работаем в линейном порядке по $\left.\partial_{i} \partial_{j} \eta_{*}\right)$

$$
2 h^{2} \chi_{\mathrm{c}}^{2}=\frac{2}{\left(\eta_{*}(\mathbf{x})-\eta\right)^{2}}+\frac{2}{3} \frac{\partial_{i} \partial_{i} \eta_{*}}{\eta_{*}-\eta} .
$$

Заметим, что можно считать $\eta_{*}=$ const в знаменателе второго члена, так как мы пренебрегаем поправками порядка $\partial_{i} \partial_{j} \eta_{*} \cdot \partial_{k} \eta_{*}$.

Получим теперь решение уравнения (7) в первом порядке по $\partial_{i} \partial_{j} \eta_{*}$. Начальное условие по-прежнему дается в (10). Будем искать решение в следующем виде:

$$
\delta \chi_{2}^{(-)}(\mathbf{k}, \mathbf{x}, \eta)=e^{i \mathbf{k x}-i k \eta_{*}(\mathbf{x})-i \mathbf{k v}\left[\eta_{*}(\mathbf{x})-\eta\right]}\left[F\left(q, \eta_{*}(\mathbf{x})-\eta\right)+F^{(2)}\left(q, \eta_{*}(\mathbf{x})-\eta\right)\right],
$$

где лидирующий вклад $F$ по-прежнему определен в $(12)$, а функция $F^{(2)}$ пропорциональна $\partial_{i} \partial_{j} \eta_{*}$. Разлагая уравнение $(7)$ по $\partial_{i} \partial_{j} \eta_{*}$, получим в линейном порядке

$$
F^{(2) \prime \prime}+k^{2} F^{(2)}-\frac{2}{\xi^{2}} F^{(2)}=\partial_{i} \partial_{i} \eta_{*} \cdot S+k_{i} k_{j} \partial_{i} \partial_{j} \eta_{*} \cdot T,
$$

где $\xi=\eta_{*}-\eta$,

$$
\begin{aligned}
& S=-i k F+\frac{\partial F(k, \xi)}{\partial \xi}+\frac{2}{3 \xi} F \\
& T=-2 F \xi-2 i \frac{\partial F(k, \xi)}{\partial k}=-\frac{2}{k^{2} \xi} e^{i k \xi} .
\end{aligned}
$$

Так как мы пренебрегаем вкладами порядка $\partial_{i} \partial_{j} \eta_{*} \cdot \partial_{k} \eta_{*}$, после вычисления правой части уравнения (35) мы положим $\mathbf{q}=\mathbf{k}$ и $\mathbf{v}=0$. Заметим, что последнее слагаемое в (36) возникает из поправки к фону (см. (34)) и что последнее выражение в (37) получено из явного вида функции Ханкеля $H_{3 / 2}^{(1)}$.

Решение $F^{(2)}$ должно исчезать при $\eta_{*}-\eta \rightarrow \infty$. Поэтому оно может быть получено с помощью запаздывающей функции Грина (напомним, что $\xi^{\prime}>\xi$ соответствует $\left.\eta^{\prime}<\eta\right)$

$$
G\left(\xi, \xi^{\prime}\right)=\frac{\pi \sqrt{\xi \xi^{\prime}}}{2} \Theta\left(\xi^{\prime}-\xi\right)\left[J_{3 / 2}(k \xi) N_{3 / 2}\left(k \xi^{\prime}\right)-N_{3 / 2}(k \xi) J_{3 / 2}\left(k \xi^{\prime}\right)\right]
$$

где $J_{3 / 2}$ и $N_{3 / 2}-$ функции Бесселя. А именно,

$$
F^{(2) T}(\xi)=\int_{\xi}^{\infty} d \xi^{\prime} G\left(\xi, \xi^{\prime}\right)\left[\partial_{i} \partial_{i} \eta_{*} \cdot S\left(\xi^{\prime}\right)+k_{i} k_{j} \partial_{i} \partial_{j} \eta_{*} \cdot T\left(\xi^{\prime}\right)\right] .
$$

Нас интересует поведение решения в "загоризонтном" режиме, $k \xi \rightarrow 0$. Поскольку наиболее сингулярное поведение $S$ и $T$ при малых $\xi$ есть $\xi^{-2}$, первый член в квадратных скобках в $(38)$ при $\xi \rightarrow 0$ дает конечный вклад в интеграл (39), тогда как второй член имеет порядок $F^{(2)}(\xi) \propto \xi^{-1}$. Следовательно, при малых $\xi$ поведение поправки $F^{(2)}(\xi)$ такое же, как и лидирующего вклада $F(\xi)$, поэтому возмущения 
фазы $\delta \theta$, включая поправки, постоянны в “загоризонтном" режиме, что является независимой проверкой нашего подхода.

Так как мы считаем тензор $\partial_{i} \partial_{j} \eta_{*}$ постоянным в видимой Вселенной, спектр мощности содержит линейную по $\partial_{i} \partial_{j} \eta_{*}$ часть ${ }^{5)}$. Этот линейный вклад возникает из интерференции $F^{(2)}$ и $F$. В “загоризонтном" режиме функция $F$ является чисто мнимой (см. (13)). Поэтому мы будем интересоваться поведением мнимой части $F^{(2)}$ при $\xi \rightarrow 0$. Последнее легко найти. Именно, для $T$-вклада в $(39)$ напишем

$$
\begin{aligned}
\left.\operatorname{Im} F^{(2) T}\right|_{\xi \rightarrow 0} & =-\frac{\pi \sqrt{\xi}}{2} N_{3 / 2}(k \xi) \int_{0}^{\infty} d \xi^{\prime} \sqrt{\xi^{\prime}} J_{3 / 2}\left(k \xi^{\prime}\right)\left(-\frac{2 \sin k \xi^{\prime}}{k^{2} \xi^{\prime}}\right) k_{i} k_{j} \partial_{i} \partial_{j} \eta_{*}= \\
& =-\frac{\pi}{2} \frac{1}{k^{2} \xi} \frac{k_{i} k_{j}}{k^{2}} \partial_{i} \partial_{j} \eta_{*} .
\end{aligned}
$$

Выполняя подобные вычисления для $S$-вклада в $(39)$, получаем в "загоризонтном" режиме

$$
F+F^{(2)}=\frac{i}{q\left(\eta_{*}-\eta\right)}\left(1-\frac{\pi}{2 k} \frac{k_{i} k_{j}}{k^{2}} \partial_{i} \partial_{j} \eta_{*}+\frac{\pi}{6 k} \partial_{i} \partial_{i} \eta_{*}\right) .
$$

Два нетривиальных члена в круглых скобках дают поправку в спектр мощности возмущений фазы, возникающих благодаря наличию радиальных мод, длины волн которых превышают хаббловский размер. Эта же поправка присуща и адиабатическим возмущениям, так что мы находим окончательно

$$
\mathcal{P}_{\zeta}=A_{\zeta}\left[1-\frac{\pi}{k} \frac{k_{i} k_{j}}{k^{2}}\left(\partial_{i} \partial_{j} \eta_{*}-\frac{1}{3} \delta_{i j} \partial_{k} \partial_{k} \eta_{*}\right)\right],
$$

где в рамках нашего приближения адиабатическая амплитуда $A_{\zeta}$ не зависит от $k$. Примечательно, что угловое усреднение поправки дает ноль, так что мы имеем дело именно со статистической анизотропией.

Ни амплитуда, ни точная форма тензора $\partial_{i} \partial_{j} \eta_{*}-(1 / 3) \delta_{i j} \partial_{k} \partial_{k} \eta_{*}$ не могут быть однозначно предсказаны из-за космической неопределенности. Чтобы оценить величину статистической анизотропии, рассмотрим дисперсию

$$
\left\langle\left(\partial \partial \eta_{*}\right)^{2}\right\rangle \equiv\left\langle\left(\partial_{i} \partial_{j} \eta_{*}-\frac{1}{3} \delta_{i j} \partial_{k} \partial_{k} \eta_{*}\right)\left(\partial_{i} \partial_{j} \eta_{*}-\frac{1}{3} \delta_{i j} \partial_{k} \partial_{k} \eta_{*}\right)\right\rangle_{k \lesssim H_{0}},
$$

где обозначение $\langle\cdot\rangle_{k \lesssim H_{0}}$ отражает тот факт, что мы приняли во внимание только те моды, современные длины волн которых превышают настоящий хаббловский размер. Используя (19), получаем

$$
\left\langle\left(\partial \partial \eta_{*}\right)^{2}\right\rangle=\frac{9 h^{2}}{16 \pi^{2}} \int_{k \lesssim H_{0}} \frac{d k}{k^{5}} \frac{2 k^{4}}{3} \simeq \frac{3 h^{2}}{4 \pi^{2}} H_{0}^{2} .
$$

Таким образом, мы приходим к первому нетривиальному вкладу в (4). Высшие поправки по градиентному разложению дают вклады в статистическую анизотропию, подавленные дополнительными факторами $H_{0} / k$.

\footnotetext{
5) Если бы мы рассматривали возмущения $\delta \eta_{*}$, длины волн которых много меньше современного хаббловского размера, то необходимо было бы выполнить усреднение по ансамблю. В этом случае линейная часть будет равна нулю.
} 
4.2. Порядок $h^{2}$ : вклад от глубоких ИК-мод. Вернемся к рассмотрению статистической анизотропии во втором порядке по $h$. Главный вклад в этом порядке пропорционален $\partial_{i} \eta_{*} \partial_{j} \eta_{*} \equiv v_{i} v_{j}$. Действительно, общий сдвиг времени не имеет отношения к делу, тогда как члены, включающие комбинации высших производных, подобные $\partial_{i} \partial_{j} \eta_{*} \partial_{i} \eta_{*}$, подавлены степенями $H_{0} / k(c p .(40),(41))$. Более того, "скорость" v усилена, хотя и только логарифмически, глубокими ИК-эффектами (см. (20)). Следовательно, для того чтобы выделить главный вклад в статистическую анизотропию в порядке $h^{2}$, мы будем использовать два члена в разложении по производным, явно указанным в (21).

Поскольку мы более не пренебрегаем членами порядка $\mathbf{v}^{2}$, функция (24) более не является решением уравнения (5). Вместо этого, сохраняя только два члена в градиентном разложении, получим решение

$$
\chi_{\mathrm{c}}=\frac{1}{h \gamma\left[\eta_{*}(0)-\eta-\mathbf{v x}\right]} .
$$

Таким образом, мы будем изучать решения уравнения (7) на этом фоне. Легко убедиться, что решением, удовлетворяющим начальному условию (10), является

$$
\delta \chi_{2}^{(-)}(\mathbf{k}, \mathbf{x}, \eta)=e^{i q_{||} \gamma\left(x_{||}+v \eta\right)+i \mathbf{q}^{\perp} \mathbf{x}^{\perp}-i q \gamma \eta_{*}(0)} F\left[q, \gamma\left(\eta_{*}(0)-\eta-\mathbf{v x}\right)\right],
$$

где лоренц-преобразованный импульс есть, как обычно, $q_{\|}=\gamma\left(k_{\|}+k v\right), \mathbf{q}^{\perp}=\mathbf{k}^{\perp}$, $q=\gamma\left(k+k_{\|} v\right), \gamma=\left(1-v^{2}\right)^{-1 / 2}$; обозначения $\|$ и $\perp$ соответствуют компонентам, параллельным и ортогональным к v. Интерпретация этого решения снова заключается в том, что оно получено путем преобразования Лоренца из решения (11).

В соответствии с обсуждаемым здесь сценарием возмущения фазы $\delta \theta$ замораживаются на гиперповерхности

$$
\eta=\eta_{*}(0)-\mathbf{v x} \equiv \eta_{*}(0)-v x_{\|},
$$

после чего они остаются постоянными во времени $\eta$. Из выражений (42) и (43) следует, что на этой гиперповерхности и позже возмущения фазы имеют вид

$$
\delta \theta(\mathbf{x})=\int \frac{d^{3} q}{\sqrt{q}} \frac{h}{4 \pi^{3 / 2} q} e^{i \gamma^{-1} q_{||} x_{||}+i \mathbf{q}^{\perp} \mathbf{x}^{\perp}} \hat{\mathcal{A}}_{\mathbf{q}}+\text { э.c. },
$$

где операторы $\hat{\mathcal{A}}_{\mathbf{q}}$ определены в $(30)$ и подчиняются стандартным коммутационным соотношениям и мы опустили не имеющий отношения к делу постоянный фазовый множитель. Мы видим, что в порядке $\mathbf{v}^{2}$ эффект глубоких ИК-мод не исчезает: он содержится в факторе $\gamma^{-1}$ в первом члене в показателе экспоненты. Ясно, что импульс возмущений, обозначенный как q, в действительности равен

$$
\mathbf{p}=\left(\gamma^{-1} q_{\| \mid}, \mathbf{q}^{\perp}\right)
$$

Соответственно спектр мощности (если опустить поправки, обсуждаемые в п. 4.1) имеет вид

$$
\mathcal{P}_{\delta \theta}(\mathbf{p}) \frac{d^{3} p}{4 \pi p^{3}}=\frac{h^{2}}{16 \pi^{3}} \frac{\gamma d^{3} p}{\left[\left(\gamma p_{\|}\right)^{2}+\left(\mathbf{p}^{\perp}\right)^{2}\right]^{3 / 2}} .
$$


Этот же спектр мощности характеризует адиабатические возмущения в нашем сценарии. Изменяя обозначение $\mathbf{p} \rightarrow \mathbf{k}$, мы окончательно получаем

$$
\mathcal{P}_{\zeta}(\mathbf{k})=A_{\zeta} \frac{p^{3}}{\left[\left(\gamma k_{||}\right)^{2}+\left(\mathbf{k}^{\perp}\right)^{2}\right]^{3 / 2}}=A_{\zeta}\left(1-\frac{3}{2} \frac{(\mathbf{k v})^{2}}{k^{2}}\right) .
$$

Таким образом, мы приходим к последнему члену в спектре (4). Как и прежде, ни направление вектора $\mathbf{v}$, ни его длина не могут быть определены из-за космической неопределенности; напомним, однако, что величина $|\mathbf{v}|$, а следовательно, и параметр $c_{2}$ в (4) логарифмически усилены благодаря ИК-эффектам (см. (20)).

В случае псевдо-намбу-голдстоуновского механизма конвертации возмущений фазы в адиабатические возмущения оба члена в выражении для адиабатической анизотропии (4) малы в силу ограничения (15). С другой стороны, эффект может быть значительным в случае механизма модулированного распада.

\section{5. ЗАКЛЮЧЕНИЕ}

В заключение мы сделаем несколько замечаний.

Во-первых, предложенный механизм генерации адиабатических возмущений может работать в любом космологическом сценарии, решающем проблему горизонта в теории горячего Большого взрыва, включая инфляцию, циклический сценарий, сценарий с отскоком, пред-Большой взрыв и т.д. В некоторых из этих сценариев (например, сценарий с отскоком) предположение, что возмущения фазы являются загоризонтными в обычном смысле в конце стадии конформного скатывания, может быть неочевидным. Было бы интересно изучить также противоположный случай, в котором фаза эволюционирует некоторое время после стадии конформного скатывания.

Во-вторых, мы сконцентрировались в этой статье на влиянии радиальных ИК-мод и применили разложение по производным. Однако при использовании полученных таким образом выражений, подобных (28), необходимо проявлять осторожность. Непосредственное использование (28) даст, например, ненулевой одновременный коммутатор $[\theta(\mathbf{x}), \theta(\mathbf{y})]$, что очевидно неправильно. Дело в том, что формула (28) справедлива в приближении $\mathbf{v}=$ const; с учетом этого одновременный коммутатор становится равным нулю, как и должно быть.

Наконец, в-третьих, нелинейность уравнений поля порождает существенную негауссовость возмущений фазы и, как следствие, адиабатических возмущений. Негауссовость возникает в порядке $O\left(h^{2}\right)$ и может быть значительна при достаточно большой константе связи $h$. Стоит отметить, что в нашей модели форма негауссовости достаточно необычна. В противоположность многим другим случаям трехточечная функция равна нулю, тогда как четырехточечная корреляционная функция возмущений $\delta \theta$ (и, следовательно, адиабатических возмущений) включает двухточечный коррелятор независимого гауссового поля $\delta \eta_{*}$. Ввиду результата, полученного в разделе 3 , корреляционные функции $\delta \theta$ должны быть ИК-конечными, по крайней мере в порядке $O\left(h^{2}\right)$. Этот результат показывает также, что анализ негауссовости выходит за рамки градиентного разложения. Поэтому мы оставляем эту проблему для будущих исследований. 
Благодарности. Авторы благодарны А. Барвинскому, А. Викману, С. Дубовскому, Д. Горбунову, Е. Комацо, С. Миронову, В. Муханову, С. Мукохояме, М. Осипову, С. Рамазанову и А. Фролову за плодотворные обсуждения. Мы благодарны за гостеприимство организаторам Yukawa International Seminar "Gravity and Cosmology", где была выполнена часть этой работы. Эта работа была частично поддержана РФФИ (грант № 08-02-00473), Федеральным агентством по науке и инновациям (государственный контракт 02.740.11.0244) и Программой поддержки ведущих научных школ (грант НШ-5525.2010.2). Работа М. В. Либанова была также частично поддержана фондом "Династия".

\section{Список литературы}

[1] E. Komatsu, K. M. Smith, J. Dunkley et al., Astrophys. J. Suppl., 192:2 (2011), 18, arXiv: 1001.4538 .

[2] А. А. Старобинский, Писъма в ЖЭЭТФ, 30:11 (1979), 719-722; Phys. Lett. B, 91:1 (1980), 99-102; A. H. Guth, Phys. Rev. D, 23:2 (1981), 347-356; A. D. Linde, Phys. Lett. B, 108:6 (1982), 389-393; 129:3-4 (1983), 177-181; A. Albrecht, P. J. Steinhardt, Phys. Rev. Lett., 48:17 (1982), 1220-1223.

[3] В. Ф. Муханов, Г. В. Чибисов, Писъма в ЖЭЭФ, 33:10 (1981), 549-552; S. W. Hawking, Phys. Lett. B, 115:4 (1982), 295-297; A. A. Starobinsky, Phys. Lett. B, 117:3-4 (1982), 175-178; A. H. Guth, S.-Y. Pi, Phys. Rev. Lett., 49:15 (1982), 1110-1113; J. M. Bardeen, P. J. Steinhardt, M. S. Turner, Phys. Rev. D, 28:4 (1983), 679-693.

[4] J. Khoury, B. A. Ovrut, P. J. Steinhardt, N. Turok, Phys. Rev. D, 64:12 (2001), 123522, 24 pp., arXiv: hep-th/0103239; J. Khoury, B. A. Ovrut, N. Seiberg, P. J. Steinhardt, N. Turok, Phys. Rev. D, 65:8 (2002), 086007, 8 pp., arXiv: hep-th/0108187.

[5] P. Creminelli, M. A. Luty, A. Nicolis, L. Senatore, JHEP, 12 (2006), 080, 30 pp., arXiv: hep-th/0606090.

[6] J. L. Lehners, P. McFadden, N. Turok, P. J. Steinhardt, Phys. Rev. D, 76:10 (2007), 103501, 10 pp., arXiv: hep-th/0702153; E. I. Buchbinder, J. Khoury, B. A. Ovrut, Phys. Rev. D, 76:12 (2007), 123503, 18 pp., arXiv: hep-th/0702154; P. Creminelli, L. Senatore, JCAP, 11 (2007), 010, 22 pp., arXiv: hep-th/0702165.

[7] A. Notari, A. Riotto, Nucl. Phys. B, 644:1-2 (2002), 371-382, arXiv: hep-th/0205019; F. Di Marco, F. Finelli, R. Brandenberger, Phys. Rev. D, 67:6 (2003), 063512, 10 pp., arXiv: astro-ph/0211276.

[8] D. Wands, Phys. Rev. D, 60:2 (1999), 023507, 4 pp., arXiv: gr-qc/9809062; F. Finelli, R. Brandenberger, Phys. Rev. D, 65:10 (2002), 103522, 8 pp., arXiv: hep-th/0112249; L. E. Allen, D. Wands, Phys. Rev. D, 70:6 (2004), 063515, 18 pp., arXiv: astro-ph/0404441; R. H. Brandenberger, Cosmology of the very early universe, arXiv: 1003.1745.

[9] S. Mukohyama, JCAP, 06 (2009), 001, 8 pp., arXiv: 0904.2190.

[10] P. Creminelli, A. Nicolis, E. Trincherini, JCAP, 11 (2010), 021, 25 pp., arXiv: 1007.0027.

[11] V.A. Rubakov, JCAP, 09 (2009), 030, 13 pp., arXiv: 0906.3693.

[12] J. K. Erickson, D. H. Wesley, P. J. Steinhardt, N. Turok, Phys. Rev. D, 69:6 (2004), 063514, 11 pp., arXiv: hep-th/0312009; D. Garfinkle, W. C. Lim, F. Pretorius, P. J. Steinhardt, Phys. Rev. D, 78:8 (2008), 083537, 8 pp., arXiv: 0808.0542.

[13] J. L. Lehners, Phys. Rept., 465:6 (2008), 223-263, arXiv: 0806.1245.

[14] V. A. Rubakov, M. A. Osipov, Писъма в ЖЖЭТФ, 93:2 (2011), 56-59, arXiv: 1007.3417.

[15] L. Ackerman, S. M. Carroll, M. B. Wise, Phys. Rev. D, 75:8 (2007), 083502, 7 pp.; Erratum, 80:6 (2009), 069901, 1 pp., arXiv: astro-ph/0701357; A. R. Pullen, M. Kamionkowski, Phys. Rev. D, 76:10 (2007), 103529, 8 pp., arXiv: 0709.1144. 
[16] M.-A. Watanabe, S. Kanno, J. Soda, Phys. Rev. Lett., 102:19 (2009), 191302, 4 pp., arXiv: 0902.2833; Prog. Theor. Phys., 123:6 (2010), 1041-1068, arXiv: 1003.0056; T.R. Dulaney, M. I. Gresham, Phys. Rev. D, 81:10 (2010), 103532, 20 pp., arXiv: 1001.2301; A. E. Gümrükçüoğlu, B. Himmetoglu, M. Peloso, Phys. Rev. D, 81:6 (2010), 063528, 16 pp., arXiv: 1001.4088 .

[17] A. E. Gümrükçüoğlu, C. R. Contaldi, M. Peloso, "CMB anomalies from relic anisotropy", Proceedings of the XI Marcel Grossmann Meeting on General Relativity (23-29 July 2006, Berlin), eds. H. Kleinert, R. T. Jantzen, R. Ruffini, World Scientific, Singapore, 2008, arXiv: astro-ph/0608405; JCAP, 11 (2007), 005, 35 pp., arXiv: 0707.4179.

[18] D. Polarski, A. A. Starobinsky, Class. Quant. Grav., 13:3 (1996), 377-391, arXiv: gr-qc/9504030.

[19] A.D. Linde, V.F. Mukhanov, Phys. Rev. D, 56:2 (1997), R535-R539, arXiv: astro-ph/9610219; K. Enqvist, M.S. Sloth, Nucl. Phys. B, 626:1-2 (2002), 395-409, arXiv: hep-ph/0109214; D. H. Lyth, D. Wands, Phys. Lett. B, 524:1-2 (2002), 5-14, arXiv: hep-ph/0110002; T. Moroi, T. Takahashi, Phys. Lett. B, 522:3-4 (2001), 215-221; Erratum, 539:3-4 (2002), 303-303, arXiv: hep-ph/0110096.

[20] K. Dimopoulos, D. H. Lyth, A. Notari, A. Riotto, JHEP, 07 (2003), 053, 24 pp., arXiv: hep-ph/0304050.

[21] G. Dvali, A. Gruzinov, M. Zaldarriaga, Phys. Rev. D, 69:2 (2004), 023505, 6 pp., arXiv: astro-ph/0303591; L. Kofman, Probing string theory with modulated cosmological fluctuations, arXiv: astro-ph/0303614.

[22] G. Dvali, A. Gruzinov, M. Zaldarriaga, Phys. Rev. D, 69:8 (2004), 083505, 5 pp., arXiv: astro-ph/0305548.

[23] F. Vernizzi, Phys. Rev. D, 69:8 (2004), 083526, 12 pp., arXiv: astro-ph/0311167. 